\title{
Elucidating feed-forward apoptosis signatures in breast cancer datasets: Higher FOS expression associated with a better outcome
}

\author{
DIANA A. FISLER ${ }^{1}$, DHIRAJ SIKARIA ${ }^{1}$, JOHN M. YAVORSKI $^{1}$, YAPING N. TU $^{1}$ and GEORGE BLANCK ${ }^{1,2}$ \\ ${ }^{1}$ Department of Molecular Medicine, Morsani College of Medicine, University of South Florida; \\ ${ }^{2}$ Immunology Program, H. Lee Moffitt Cancer Center, Tampa, FL 33612, USA
}

Received February 20, 2018; Accepted May 22, 2018

DOI: $10.3892 /$ ol.2018.8957

\begin{abstract}
Overstimulation of pro-proliferative pathways and high level expression of pro-proliferative transcription factors (TFs) can lead to apoptosis. This is likely due to TF binding sites for pro-proliferative TFs common to pro-proliferative and pro-apoptosis-effector genes. Certain clinical datasets have indicated that molecular markers associated with higher proliferation rates lead to improved outcomes for patients with cancer. These observations have been extensively assessed on a general basis, however there has been little work dissecting feed-forward apoptosis signaling pathways that may represent specific distinctions between a pro-proliferative mechanism and a pro-apoptotic mechanism in samples from patients with cancer. Using The Cancer Genome Atlas datasets and bioinformatic approaches, the present study reports that higher FOS expression levels, along with higher FOS target apoptosis-effector gene expression, is associated with an increased survival, while higher POU2F1 expression is associated with a reduced survival (average difference of 25.9 months survival). In summary, in the datasets examined FOS represents an apoptosis-driver and high POU2F1 represents a driver mechanism for cancer development.
\end{abstract}

\section{Introduction}

There are several, distinct pathways that can drive cells into apoptosis. For example, cross-linking of major histocompatibility class II molecules apparently represents an S-phase independent mechanism of apoptosis (1). Another, basic pathway involves the over-accumulation of what would normally be pro-proliferative transcription factors (TFs), for example, E2F1 (2). This over-accumulation presumably occurs

Correspondence to: Professor George Blanck, Department of Molecular Medicine, Morsani College of Medicine, University of South Florida, 12901 Bruce B. Downs Boulevard, Tampa, FL 33612, USA

E-mail: gblanck@health.usf.edu

Key words: apoptosis-driver, cancer-driver, apoptosis-effector genes, breast cancer, FOS, UQCRC2, GZMA in situations where S-phase is impeded due to a defect in the cell or due to lack of metabolites or proteins needed for a complete progression through S-phase. The over-accumulation then leads to occupancy of pro-apoptosis-effector genes, by the pro-proliferative TFs. This feed forward process of apoptosis is exemplified by over-activation of the T-cell receptor signaling pathway in deletion of self-reactive T-cells in the thymus (3) and by treatment of cells with interferon- $\gamma$ (4), which is pro-proliferative at lower concentrations and pro-apoptotic at higher concentrations. The importance of a specific pro-proliferative TF in apoptosis was elegantly demonstrated many years ago by unexpected tumor development in mice lacking E2F1 (5-7), a classic pro-proliferative TF that stimulates histone gene and dihydrofolate reductase gene expression $(8,9)$, in preparation for S-phase. POU2F1 and RB1 have been shown to regulate the interferon- $\gamma$ transition from stimulation of proliferation to stimulation of apoptosis $(4,10)$.

There are several possible and not necessarily mutually exclusive mechanisms to explain the basis of feed forward apoptosis (FFA). One possibility is based on the fact that apoptosis-effector genes are generally smaller than proliferation-effector genes $(11,12)$, leading to the proposal that the occupancy of the apoptosis-effector genes is simply due to stochastic processes that favor initial occupancy of proliferation-effector genes, and then upon reaching a high enough intra-cellular concentration, lead to occupancy of the apoptosis-effector genes $(12,13)$. A second possibility, established via an E2F1 model system $(14,15)$, indicates that E2F1 binding partners will change in the transition from E2F1 occupancy of proliferation-effector genes to E2F1 occupancy of apoptosis-effector genes.

In the cancer patient setting, evidence of feed forward apoptosis has been detected in several ways. First, MYC amplification in neuroblastoma represents a better outcome, with conventional therapy, unless CASP8 is absent (16). And, using The Cancer Genome Atlas (TCGA) data, we have recently reported that an increased number of oncoprotein or tumor suppressor protein mutations in stomach adenocarcinoma represents a better outcome (17). It is clear that cancer progression represents a balance of proliferation-effector and apoptosis-effector gene expression, such that proliferation overcomes apoptosis in case of terminal cancer $(18,19)$. This has led to the question of whether individual pro-proliferative TFs can be classified as supporting apoptosis, presumably despite 
an overwhelming impact of other, specific TFs supporting proliferation. The results presented below represent one of the first such identifications of a specific feed-forward apoptosis pathway, that is balanced by a specific but distinct pro-proliferative $\mathrm{TF}$, in a cancer patient setting.

\section{Materials and methods}

Basic approaches. RNA microarray values from the metabric breast cancer dataset representing pro-proliferative TFs (FOS, E2F1, JUN, POU2F1, MYC, YY1, STAT3, NFATC1) (20) were obtained from www.cbioportal.org. The microarray values and their associated barcodes were organized in descending order for each TF. The barcodes representing the top $20 \%$ and bottom $20 \%$ values were obtained and used as selected samples for the Kaplan-Meier (KM) survival curve analysis tool of cbioportal.org; or for the IBM Statistical Package for the Social Sciences (SPSS; IBM Corp., Armonk, NY, USA) software, exactly as described (21) to verify results using cbioportal.org web tool. The apoptosis-effector genes used in this study were obtained from a previous study (12). As previously shown (12), all apparent human apoptosis-effector genes were first obtained on the bases of keyword searches of the human genome browser database. Then, a set of 34 apoptosis-effector genes was established by inspection. From this set of 34 apoptosis-effector genes, we identified the apoptosis-effector genes with AP1 (FOS) and POU2F1-binding sites within 5,000 base pairs on either side of the gene, inclusive, using the hg19 genome browser database, with a $\mathrm{z}$-score cutoff of 2.33. The microarray values for each of the apoptosis-effector genes, with either AP1 (FOS) or POU2F1 binding sites, were obtained from cbioportal.org.

Distinct breast cancer dataset. The RNASeq values of GZMA were obtained for the TCGA-BRCA 1105 dataset, from www. cbioportal.org. The microarray data were then sorted into descending order to identify top $20 \%$ and bottom $20 \%$ GZMA RNASeq values. The averages for top $20 \%$ and bottom $20 \%$ were calculated, and associated barcodes were analyzed via KM approaches as detailed above.

GZMA methylation. The beta-score, methylation values of the TCGA-BRCA GZMA dataset were obtained from cbioportal. org and processed as previously described (22). The barcodes were sorted and organized into their respective top $20 \%$ and bottom $20 \%$ levels for statistical analyses.

GZMA RNASeq read counts. The RNASeq read counts were generated by downloading raw RNAseq files from the genome data commons, via approved NIH dbGaP project no. 6300, and summing up the reads representing the GZMA section of the genome.

Statistical analysis. The statistical data in this report are presented as correlation plots, KM analyses and differences in the means. For correlation plots, a correlation coefficient and P-value were obtained; for the KM analyses, a log-rank $\mathrm{P}$-value was obtained and used to establish significance; and for the differences in the means, as presented in the Tables, a Student's t-test was used to establish significance. Excel (version 16.12; Microsoft Corporation, Redmond, WA, USA) was used to obtain correlation coefficients and MedCalc (version 2017; MedCalc Software, Ostend, Belgium) was used to obtain the P-values for the linear correlations. The KM analyses log-rank P-values were obtained with the SPSS. For the majority of the analyses, there were 381 tumor samples representing the upper and lower quintiles in the analyses.

\section{Results}

Association of higher FOS expression with better survival and relatively high expression of FOS responsive apoptosis-effector genes. To determine whether there were expression levels of pro-proliferative TFs, that correlated or inversely correlated with overall breast cancer survival, we first obtained the microarray values for eight candidate, pro-proliferative TFs as tabulated and shown in a previous study. (12). The purpose of this determination was to identify candidate TFs, whereby one TF with a high expression level could be indicative of driving FFA, and thereby be associated with better survival; and a second TF could, with a high expression level, be associated with a worse survival. Presumably the latter TF would be driving proliferation in the absence of a functional FFA process for that TF. The microarray data were organized into groups of barcodes (samples) that represented the top $20 \%$ and bottom $20 \%$ of expression levels for each of the eight pro-proliferative TFs (12). The barcodes for those two groups, for each TF, were compared in terms of the survival rates, with the remaining barcodes in the dataset, respectively, using a KM curve, available via a cbioportal.org web tool. Results indicated that high levels of FOS were associated with a better survival and that high levels of POU2F1 were associated with a worse survival. The other six TFs did not show a statistically significant association with either survival status and were not further considered in this study (Table I).

To confirm the above indication, that high levels of FOS and POU2F1 represented opposite survival rates, the top 20\% expressers and bottom $20 \%$ expressers for each of these TFs were compared in KM analyses, with results consistent with the conclusions in the previous paragraph, namely that high FOS is associated with better survival and high POU2F1 associated with poorer survival (Fig. 1).

To determine whether a higher level of FOS expression correlated with higher apoptosis-effector gene expression, we obtained the RNA microarray values for all of the apoptosis-effector genes characterized as previously described (12) that contain an AP1 (FOS/JUN) site within 5,000 base pairs, inclusive, specifically, UQCRC2, BAD, BAX, and CRADD. We next sorted the FOS expression levels and obtained the microarray value averages for each of the AP1 site-containing apoptosis-effector genes listed above for the barcodes that represented the top $20 \%$ and bottom $20 \%$ of FOS expression. We then determined whether the top $20 \%$ and bottom $20 \%$ microarray value averages represented significant differences, in the case of each of these apoptosis-effector genes. Only UQCRC2 and CRADD represented statistically significant differences (Table II). The microarray values for UQCRC2 (in relation to FOS) represented an average of 8.212 for the top 20\% FOS expressers and 7.585 for the bottom $20 \%$ FOS expressers. The results of the CRADD analysis, for the 
Table I. P-values representing the overall survival differences for the breast cancer barcodes representing the top and bottom $20 \%$ of expression levels (microarray values) for the indicated transcription factors.

\begin{tabular}{lcc}
\hline \multirow{2}{*}{$\begin{array}{l}\text { Transcription } \\
\text { factor }\end{array}$} & \multicolumn{2}{c}{ P-value } \\
\cline { 2 - 3 } & Top 20\% & Bottom 20\% \\
\hline FOS & 0.011 (better & 0.031 (worse \\
& overall survival) & overall survival) \\
E2F1 & 0.69 & 0.39 \\
JUN & 0.10 & 0.012 \\
POU2F1 & 0.0034 (worse & 0.0027 (better \\
& overall survival) & overall survival) \\
MYC & 0.82 & 0.14 \\
YY1 & 0.13 & 0.42 \\
STAT3 & 0.37 & $\mathrm{NS}$ \\
NFATC1 & 0.64 & 0.25 \\
\hline
\end{tabular}

NS, not significant; FOS, Fos proto-oncogene AP-1 transcription factor subunit; E2F1, E2F transcription factor 1; JUN, Jun proto-oncogene AP-1 transcription factor subunit; POU2F1, POU class 2 homeobox 1; MYC, Myc proto-oncogene BHLH transcription factor; YY1, YY1 transcription factor; STAT3, signal transducer and activator of transcription 3; NFATC1, nuclear factor of activated T cells 1 .
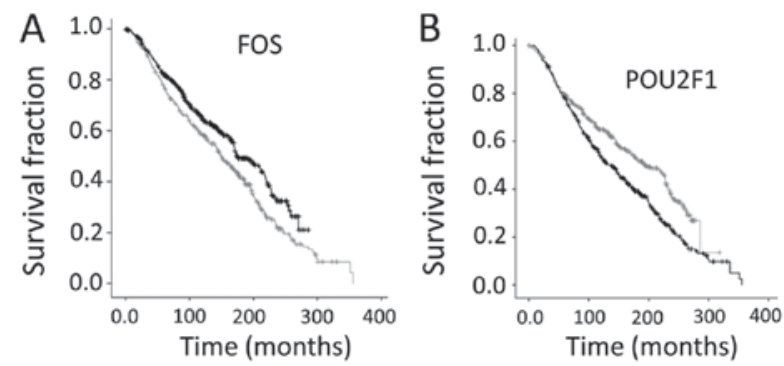

Figure 1. Kaplan-Meier curves for barcodes with high and low expression of FOS and POU2F1. Top 20\% expressers for each TF is represented by the darker black curve; bottom $20 \%$ expressers for each TF is represented by the lighter gray curve. The log-rank $\mathrm{P}$-values for the differences in the survival rates for (A) FOS and for (B) $\mathrm{POU} 2 \mathrm{~F} 1$ were $\mathrm{P}=0.003514$ and $\mathrm{P}=0.000134$, respectively. For FOS, the mean survival difference is 15.3 months. For POU2F1, 25.9 months. TF, transcription factor; FOS, Fos proto-oncogene AP-1 transcription factor subunit; POU2F1, POU class 2 homeobox 1.
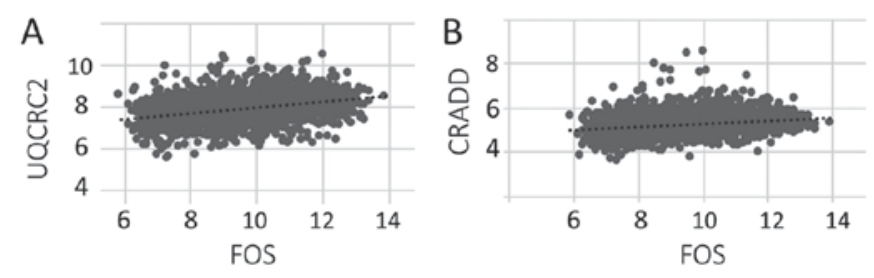

Figure 2. Scatter plots of microarray values for FOS and the two pro-apoptosis-effector genes with FOS binding sites, UQCRC2 and CRADD. (A) The correlation coefficient plot of FOS vs. UQCRC2 are positively correlated (0.32) with a linear correlation of $\mathrm{r}^{2}=0.10$. (B) Correlation coefficient plot of FOS vs. CRADD is positively correlated (0.25) with a linear correlation of $\mathrm{r}^{2}=0.06$. $\mathrm{P}<0.0001$ for each. FOS, Fos proto-oncogene AP-1 transcription factor subunit; UQCRC2, ubiquinol-cytochrome $\mathrm{C}$ reductase core protein 2 CRADD, CASP2 and RIPK1 domain containing adaptor with death domain.
Table II. Comparison of microarray value averages for apoptosis-effector genes UQCRC2 and CRADD, for the FOS high and low expressers in the breast cancer dataset.

\begin{tabular}{lccc}
\hline $\begin{array}{l}\text { Effector } \\
\text { gene }\end{array}$ & $\begin{array}{c}\text { Top 20\% FOS } \\
\text { expressers }\end{array}$ & $\begin{array}{c}\text { Bottom 20\% } \\
\text { FOS expressers }\end{array}$ & $\begin{array}{c}\text { P-value } \\
\text { (top vs. bottom) }\end{array}$ \\
\hline UQCRC2 & 8.212 & 7.585 & $<0.0001$ \\
CRADD & 7.385 & 7.039 & $<0.0001$ \\
\hline
\end{tabular}

UQCRC2, ubiquinol-cytochrome $\mathrm{C}$ reductase core protein 2; CRADD, CASP2 and RIPK1 domain containing adaptor with death domain; FOS, Fos proto-oncogene AP-1 transcription factor subunit.

categories of top $20 \%$ and bottom $20 \%$ FOS expressers, represented an average of 7.385 in the top $20 \%$ FOS expressers and an average of 7.039 for the bottom $20 \%$ FOS expressers.

To confirm the correlation of expression levels between FOS and UQCRC2 or CRADD, we determined the Pearson correlation coefficient for the FOS microarray values and the apoptosis-effector gene RNA microarray values. Both the UQCRC2 (Fig. 2A) and CRADD (Fig. 2B) apoptosis-effector genes showed a linear correlation with the (entire) set of FOS microarray values. Thus, the correlation data were consistent with the above described indication that the barcodes representing the top $20 \%$ FOS expressers and bottom $20 \%$ of FOS expressers also represented statistically significant differences in the expression of UQCRC2 and CRADD.

High levels of POU2F1 are generally not associated with high levels of POU2F1 responsive apoptosis-effector genes. As noted above (Table I; Fig. 1B), higher levels of POU2F1 microarray values are associated with statistically significant worse survival. To determine whether apoptosis-effector genes with POU2F1 binding sites represented either more or less expression when POU2F1 levels were high, we obtained the microarray values for the following POU2F1-site containing, apoptosis-effector genes: UQCRC2, GZMA,CRADD, CHEK1, CASP5, CASP3, and COX7B2. We then sorted the barcodes based on top $20 \%$ and bottom $20 \%$ POU2F1 expressers. We next obtained the microarray values for the above indicated apoptosis-effector genes (with the POU2F1-binding sites), for the top $20 \%$ and bottom $20 \%$ POU2F1 expression categories, respectfully (Table III). All of the apoptosis-effector genes indicated statistically significant differences in expression levels for the upper and lower POU2F1 expression categories with the exception of COX7B2. In the case of COX7B2, the microarray values for top and bottom POU2F1 expressers showed no significant differences. In these POU2F1-site containing genes, the barcodes at the highest level of POU2F1 expression had lower levels of apoptosis gene expression, and the barcodes at the lowest level of POU2F1 expression had higher levels of apoptosis-effector gene expression. These results are consistent with POU2F1 as a cancer driver and a TF that is not activating what would otherwise be POU2F1-responsive, pro-apoptotic genes (Table III). The one exception to this trend was CASP3 (Table III). In addition, we determined the Pearson correlation coefficients for the entire set of microarray values for POU2F1 vs. UQCRC2, GZMA, and CRADD, respectfully 
Table III. Breast cancer expression levels of POU2F1 transcription factor binding site-containing, apoptosis-effector genes .

Transcription factor

Top 20\% POU2F1 expressers, mean microarray value
Bottom 20\% POU2F1 expressers

mean microarray value
P-value

POU2F1-independent

predictors of survival rates

$\begin{array}{llll}\text { UQCRC2 } & 7.41 & 8.38 & 0.0001 \\ \text { GZMA } & 6.84 & 7.27 & 0.0001\end{array}$

Do not associate with

differing survival rates

\begin{tabular}{llcc} 
CRADD & 6.90 & 7.45 & 0.0001 \\
CHEK1 & 6.50 & 6.68 & 0.0001 \\
CASP5 & 5.44 & 5.46 & 0.0558 \\
CASP3 & 8.67 & 8.57 & 0.0004 \\
COX7B2 & 5.41 & 5.43 & NS \\
\hline
\end{tabular}

Comparison of POU2F1 transcription factor binding site genes (12). The top $20 \%$ and bottom $20 \%$ calculated averages of the seven apoptosis-effector genes, respectively, with binding sites for POU2F1 are indicated. Of the seven, two apoptosis-effector genes show a clear distinction in worse overall survival for the top $20 \%$ expressers (of the indicated apoptosis gene) compared with the bottom $20 \%$ expressers. These two apoptosis-effector genes are TF-independent markers of survival rates. NS, not significant; POU2F1, POU class 2 homeobox 1; UQCRC2, ubiquinol-cytochrome $\mathrm{C}$ reductase core protein 2; GZMA, granzyme A; CRADD, CASP2 and RIPK1 domain containing adaptor with death domain; CHEK1, checkpoint kinase 1; CASP, caspase; COX7B2, cytochrome C oxidase subunit $7 \mathrm{~B} 2$.

(Fig. 3). These three apoptosis-effector genes were chosen for the Pearson correlation coefficient analysis because they represented the largest separation of expressions values representing the top and bottom $20 \%$ POU2F1 expressers (Table III). (Also, these three apoptosis-genes were studied in the next section as independent markers of survival.) Results from the Pearson correlation coefficient analyses (Fig. 3) were consistent with the above indicated distinctions based on the analyses of the top $20 \%$ and bottom $20 \%$ of POU2F1 expressers (Table III).

Apoptosis-effector genes expression levels as independent indicators of survival rates. To determine whether the expression levels of the apoptosis-effector genes could be independent indicators of survival distinctions, we sorted the barcodes based on the microarray values for each apoptosis-effector gene, without regard for the expression of any of the TFs. Barcodes representing the top $20 \%$ of UQCRC2 microarray expression levels displayed a greater overall survival rate $(\mathrm{P}=0.0037)$ when compared to all remaining samples (Table IV). And, the barcodes representing the bottom $20 \%$ of UQCRC 2 microarray values displayed a worse overall survival when compared to all remaining samples $(\mathrm{P}=0.05)$ (Table IV). Likewise, barcodes representing the top $20 \%$ of GZMA microarray expression levels displayed a greater overall survival rate when compared to all remaining barcodes $(\mathrm{P}=0.012)$. And, barcodes representing the bottom $20 \%$ of GZMA microarray expression levels displayed a worse overall survival rate when compared to all remaining samples $(\mathrm{P}=0.0032)$ (Table IV). The expression levels of the remaining apoptosis-effector genes (Table III) did not indicate any independent associations with distinct survival rates.

To confirm the above indication, that UQCRC2 and GZMA represented independent correlations with survival rates, KM survival curves were created representing in each case the top
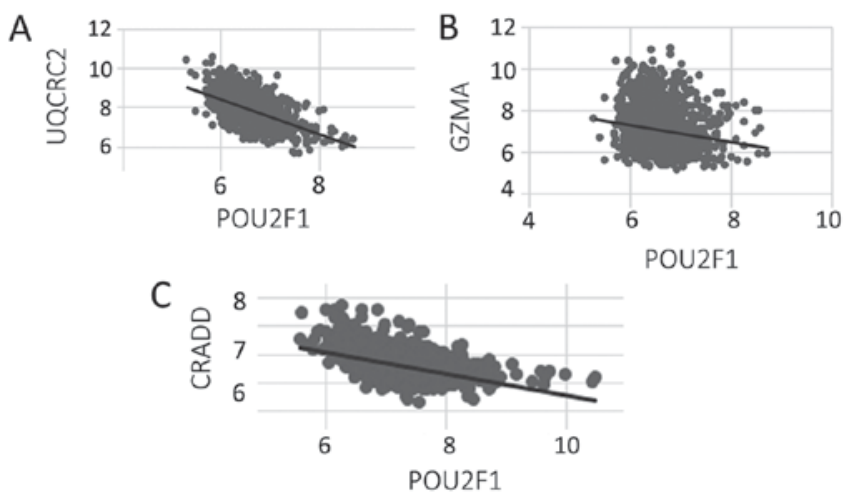

Figure 3. Scatter plots of POU2F1 microarray values vs. associated apoptosis-effector genes UQCRC2, GZMA, CRADD. The linear correlation coefficient plots of (A) POU2F1 vs. UQCRC2, (B) POU2F1 vs. GZMA and (C) POU2F1 vs. CRADD are negatively correlated, with $\mathrm{r}^{2}=0.29,0.028,0.21$, respectfully. In all three cases, $\mathrm{P}<0.00001$. POU2F1, POU class 2 homeobox 1; UQCRC2, ubiquinol-cytochrome $\mathrm{C}$ reductase core protein 2; GZMA, granzyme A; CRADD, CASP2 and RIPK1 domain containing adaptor with death domain.

$20 \%$ and bottom 20\% expressers, using the SPSS software (Fig. 4). Results indicated that higher levels of both UQCRC2 and GZMA were associated with better survival rates.

GZMA as an independent indicator for survival rate in a distinct breast cancer dataset. Overall KM survival curve of patients representing the top $20 \%$ of expressers of the apoptosis gene, GZMA, in a different breast cancer data set (TCGA-BRCA, with 1105 samples), displayed a better survival, compared with all remaining barcodes $(\mathrm{P}=0.11)$. There was a worse survival rate for the bottom $20 \%$ GZMA expressers, compared with all remaining barcodes $(\mathrm{P}=0.032)$. An additional, independently constructed KM survival curve was created to further verify the 
Table IV. Breast cancer expression levels apoptosis-effector genes used for survival rate correlations independent of the pro-proliferative transcription factors.

Top 20\% apoptosis-gene

Transcription factor expressers, mean microarray value
Bottom 20\% apoptosis-gene

expressers, mean microarray value
P-value

UQCRC2, ubiquinol-cytochrome C reductase core protein 2; GZMA, granzyme A.

Table V. Granzyme A methylation levels for The Cancer Genome Atlas-BRCA dataset.

\begin{tabular}{lccc}
\hline Data set & $\begin{array}{c}\text { Top 20\% } \\
\text { expressers }\end{array}$ & $\begin{array}{c}\text { Bottom 20\% } \\
\text { expressers }\end{array}$ & P-value \\
\hline TCGA-BRCA & 0.609 & 0.853 & $<0.0001$ \\
\hline
\end{tabular}

TCGA, The Cancer Genome Atlas.

above information that GZMA displayed significant survival differences in a second distinct breast cancer dataset (Fig. 5).

GZMA methylation. To consider a mechanistic explanation for GZMA differences in the expression levels among the TCGA barcodes, we hypothesized that GZMA methylation could lead to a repressive chromatin structure and thereby block access to POU2F1 in the cells representing the barcodes with high POU2F1 levels and relatively low levels of GZMA. Thus, we downloaded the TCGA-BRCA methylation data for GZMA and organized the barcodes into the top $20 \%$ and bottom $20 \%$ of GZMA expression levels. (The metabric study did not have methylation data.) We calculated the GZMA methylation (beta-score) of the top 20\% GZMA expressers to be 0.61 ; and calculated the GZMA methylation for the bottom $20 \%$ of the GZMA expressers to be 0.85 , representing a higher level of GZMA gene methylation and a statistically significant difference (Table V).

Raw RNAseq data verification. Because the above analysis was done with processed data, we sought to verify the final conclusion, that the apoptosis effector gene, GZMA, represented a robust biomarker for breast cancer survival. Thus, we downloaded the original RNASeq files from the genome data commons for the top and bottom $20 \%$ GZMA expressers and obtained read counts representing GZMA, i.e., hg38 reference genome position, chr5:55, 102, 648-55, 110, 252. The average number of reads for the top $20 \%$ expressers was 2698 ; the average number of reads for the bottom $20 \%$ expressers was $107(\mathrm{P}<0.0001)$.

\section{Discussion}

As indicated by the above analyses, available data are consistent with the idea that feed-forward pathways can represent specific distinctions, based on what have been traditionally considered pro-proliferative TFs, between pro-proliferative and pro-apoptotic pathways. This conclusion was also recently reached for
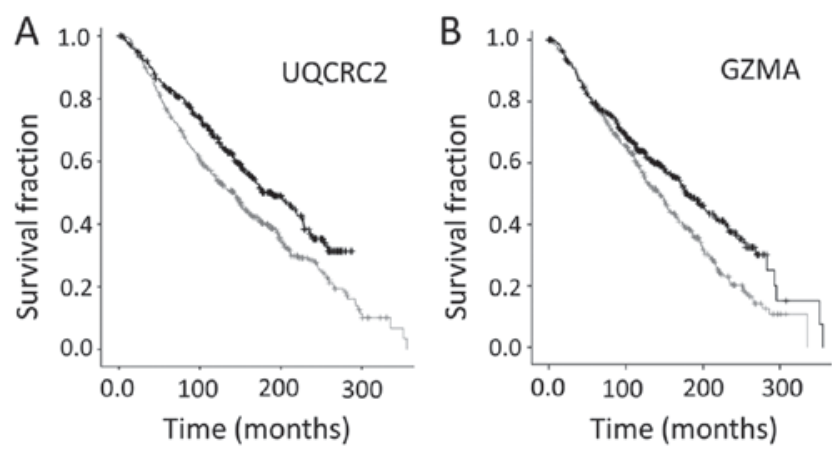

Figure 4. Kaplan-Meier curves for (A) UQCRC2 and (B) GZMA survival differences. Top $20 \%$ of the microarray values for each gene is represented by the darker black curve. Bottom $20 \%$, by the lighter gray curve. The log-rank $\mathrm{P}$-values for UQCRC2 and GZMA were $\mathrm{P}=0.000285$ and $\mathrm{P}=0.000971$, respectively. The mean difference in survival times for UQCRC2 and GZMA were 21.1 and 29.9 months, respectively. UQCRC2, ubiquinol-cytochrome C reductase core protein 2; GZMA, granzyme A.

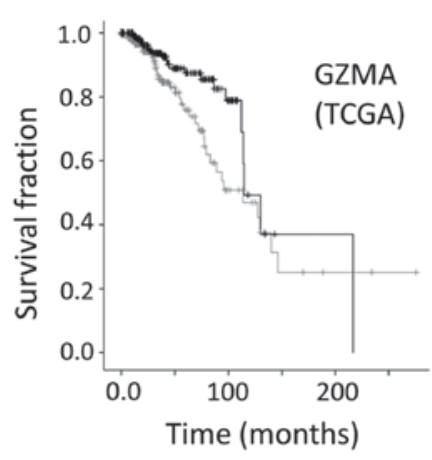

Figure 5. Kaplan-Meier curves for GZMA survival differences for the TCGA-BRCA dataset. Top $20 \%$ for the TCGA-BRCA microarray values is represented by the darker black curve. Bottom $20 \%$ is represented by the lighter gray curve. The log-rank $\mathrm{P}$-value was $\mathrm{P}=0.022$. GZMA, granzyme A; TCGA, The Cancer Genome Atlas.

lower grade glioma and squamous cell lung cancer (23), where MYC and YY1, respectively, were identified as apoptosis-drivers. These distinctions may be useful in determining a more accurate overall survival rate among cancer patients, as well as possibly assist in development of therapies. In this study, low POU2F1 barcodes represented a 25.9 month longer survival, on average.

In the case of the above breast cancer dataset, the analyses indicated that high levels of FOS were associated with better survival, and that high levels of POU2F1, as noted in the previous paragraph, were associated with worse survival outcomes. To understand the link between FOS and better survival, FOS was 
connected to higher expression of apoptosis-effector genes, thereby indicating a credible pathway for FFA, with FOS as the apoptosis-driver. In particular, the higher level of FOS expression was correlated with higher expression of the apoptosis-effector genes, CRADD and UQCRC2.

To better understand how lower levels of POU2F1 could be associated with a higher survival rate, we examined the POU2F1 relationship with its putatively responsive, apoptosis-effector genes: UQCRC2, GZMA, CRADD, CHEK1, CASP5. In all of these cases, the highest level of gene expression was associated with the lowest levels of POU2F1. As noted in Results, in two cases, there was not an inverse correlation with POU2F1 expression for the POU2F1 putatively responsive, apoptosis-effector genes. In these latter two cases, there is no explanation for the lack of an inverse correlation of expression levels, other than the possibility that the expression of the two apoptosis-effector genes does not interfere with POU2F1 as a cancer-driver due to a mechanism that is not related to transcriptional activation, for example, micro-RNA mediated, negative regulation at the level of translation. Investigations of such a possibilities will be part of future work.

While the above connections between TFs and apoptosis-effector genes indicates a mechanism whereby higher levels of what are traditionally consider pro-proliferative TFs could lead to better survival, an additional, practical issue is, biomarkers for improved for survival rates. As such, we examined apoptosis-effector gene expression for TF-independent associations with distinct survival rates, with GZMA and UQCRC2 representing the most important such biomarker candidates, including the confirmation of higher GZMA expression levels representing better overall survival rates in a second breast cancer dataset, namely the TCGA BRCA dataset. GZMA is expressed at a high level in cytotoxic T-cells (24), and none of the above data can indisputably pinpoint the source of the gene expression studied, i.e., cancer cells or micro-environment cells. Thus, it is possible that the higher level of GZMA represents a higher level of cytotoxic T-cell infiltrate that is mediating an increased level of apoptosis and better survival rates. However, there is no such specific association of the FOS-responsive UQCRC2 with immune infiltrate cells, and what data is available indicates mammary tissue and whole blood represent about the same levels of expression (genome.ucsc.edu), very different from GZMA.

In sum, the above analyses identified potential FFA signatures in breast cancer with possible insights into therapy design and likely survival rate, biomarker identification.

\section{Acknowledgements}

Not applicable.

\section{Funding}

No funding was received.

\section{Availability of data and materials}

The datasets used and/or analyzed during the current study are available from the corresponding author on reasonable request.

\section{Authors' contributions}

DAF designed the approach to testing the main hypotheses and conducted the majority of the analyses. DS conducted the raw RNAseq data verification. JMY conducted the methylation analyses and assisted with Kaplan-Meier analyses. YT contributed the analyses of the raw RNASeq files for verifications of processed data and contributed to drafting the manuscript. GB supervised the project and contributed extensively to the analysis of the results. GB also wrote and prepared the manuscript, formulated important intellectual content and critical reviewed all aspects of data collection, analysis and preparation.

\section{Ethics approval and consent to participate}

Not applicable.

\section{Consent for publication}

Not applicable.

\section{Competing interests}

The authors declare that they have no competing interests.

\section{References}

1. Truman JP, Choqueux C, Charron D and Mooney N: HLA class II molecule signal transduction leads to either apoptosis or activation via two different pathways. Cell Immunol 172: 149-157, 1996.

2. Ford SA and Blanck G: Signal persistence and amplification in cancer development and possible, related opportunities for novel therapies. Biochim Biophys Acta 1855: 18-23, 2015.

3. Macián F, Im SH, García-Cózar FJ and Rao A: T-cell anergy. Curr Opin Immunol 16: 209-216, 2004.

4. Szekeres K, Koul R, Mauro J, Lloyd M, Johnson J and Blanck G: An Oct-1-based, feed-forward mechanism of apoptosis inhibited by co-culture with Raji B-cells: Towards a model of the cancer cell/B-cell microenvironment. Exp Mol Pathol 97: 585-589, 2014.

5. Field SJ,Tsai FY,KuoF,Zubiaga AM,Kaelin WG Jr,Livingston DM, Orkin SH and Greenberg ME: E2F-1 functions in mice to promote apoptosis and suppress proliferation. Cell 85: 549-561, 1996.

6. Wu X and Levine AJ: p53 and E2F-1 cooperate to mediate apoptosis. Proc Natl Acad Sci USA 91: 3602-3606, 1994.

7. Yamasaki L, Jacks T, Bronson R, Goillot E, Harlow E and Dyson NJ: Tumor induction and tissue atrophy in mice lacking E2F-1. Cell 85: 537-548, 1996.

8. Oswald F, Dobner T and Lipp M: The E2F transcription factor activates a replication-dependent human $\mathrm{H} 2 \mathrm{~A}$ gene in early S phase of the cell cycle. Mol Cell Biol 16: 1889-1895, 1996.

9. Banerjee D, Ercikan-Abali E, Waltham M, Schnieders B, Hochhauser D, Li WW, Fan J, Gorlick R, Goker E and Bertino JR: Molecular mechanisms of resistance to antifolates, a review. Acta Biochim Pol 42: 457-464, 1995.

10. Berry DE, Lu Y, Schmidt B, Fallon PG, O'Connell C, Hu SX, $\mathrm{Xu} \mathrm{HJ}$ and Blanck G: Retinoblastoma protein inhibits IFN-gamma induced apoptosis. Oncogene 12: 1809-1819, 1996.

11. McKay BC, Becerril C, Spronck JC and Ljungman M: Ultraviolet light-induced apoptosis is associated with S-phase in primary human fibroblasts. DNA Repair (Amst) 1: 811-820, 2002.

12. Mauro JA and Blanck G: Functionally distinct gene classes as bigger or smaller transcription factor traps: A possible stochastic component to sequential gene expression programs in cancer. Gene 536: 398-406, 2014.

13. Garcia M, Mauro JA, Ramsamooj M and Blanck G: Tumor suppressor genes are larger than apoptosis-effector genes and have more regions of active chromatin: Connection to a stochastic paradigm for sequential gene expression programs. Cell Cycle 14: 2494-2500, 2015 
14. Lu H, Liang X, Issaenko OA and Hallstrom TC: Jab1/CSN5 mediates E2F dependent expression of mitotic and apoptotic but not DNA replication targets. Cell Cycle 10: 3317-3326, 2011.

15. $\mathrm{Lu} \mathrm{H}$ and Hallstrom TC: The nuclear protein UHRF2 is a direct target of the transcription factor E2F1 in the induction of apoptosis. J Biol Chem 288: 23833-4333, 2013.

16. Teitz T, Wei T, Valentine MB, Vanin EF, Grenet J, Valentine VA Behm FG, Look AT, Lahti JM and Kidd VJ: Caspase 8 is deleted or silenced preferentially in childhood neuroblastomas with amplification of MYCN. Nat Med 6: 529-535, 2000.

17. Yavorski JM and Blanck G: TCGA: Increased oncoprotein coding region mutations correlate with a greater expression of apoptosiseffector genes and a positive outcome for stomach adenocarcinoma. Cell Cycle 15: 1-7, 2016.

18. Mauro JA, Butler SN, Ramsamooj M and Blanck G: Copy number loss or silencing of apoptosis-effector genes in cancer. Gene 554: 50-57, 2015.

19. Mauro JA, Yavorski JM and Blanck G: Stratifying melanoma and breast cancer TCGA datasets on the basis of the CNV of transcription factor binding sites common to proliferation- and apoptosis-effector genes. Gene 614: 37-48, 2017.
20. Cerami E, Gao J, Dogrusoz U, Gross BE, Sumer SO, Aksoy BA, Jacobsen A, Byrne CJ, Heuer ML, Larsson E, et al: The cBio cancer genomics portal: An open platform for exploring multidimensional cancer genomics data. Cancer Discov 2: 401-404, 2012.

21. Callahan BM, Patel JS, Fawcett TJ and Blanck G: Cytoskeleton and ECM tumor mutant peptides: Increased protease sensitivities and potential consequences for the HLA class I, mutant epitope reservoir. Int J Cancer 142: 988-998, 2017.

22. Yavorski JM and Blanck G: Smoking correlates with increased cytoskeletal protein-related coding region mutations in the lung and head and neck datasets of the cancer genome atlas. Physiol Rep 4: pii: e13045, 2016.

23. Sikaria D, Tu YN, Fisler DA, Mauro JA and Blanck G: Identification of specific feed-forward apoptosis mechanisms and associated higher survival rates for low grade glioma and lung squamous cell carcinoma. J Cancer Res Clin Oncol 144: 459-468, 2018.

24. Lieberman J: Granzyme A activates another way to die. Immunol Rev 235: 93-104, 2010. 